\title{
Vasopressin 1b Receptor Knock-Out Impairs Memory for Temporal Order
}

\author{
Loren M. DeVito, ${ }^{1}$ Rachael Konigsberg, ${ }^{1}$ Christine Lykken, ${ }^{1}$ Magdalena Sauvage, ${ }^{1}$ W. Scott Young III, ${ }^{2}$ and \\ Howard Eichenbaum ${ }^{1}$ \\ ${ }^{1}$ Center for Memory and Brain, Boston University, Boston, Massachusetts 02215, and ${ }^{2}$ Section on Neural Gene Expression, National Institute of Mental \\ Health-National Institutes of Health, Department of Health and Human Services, Bethesda, Maryland 20892
}

Mice lacking a functional vasopressin $1 \mathrm{~b}$ receptor (Avpr1b) display decreased levels of aggression and social memory. Here, we used Avprlb-knock-out (Avprlb ${ }^{-I-}$ ) mice to examine whether an abnormality of this receptor results in specific cognitive deficits in the domain of hippocampal function. Avprlb ${ }^{-1-}$ mice were deficient in sociability and in detecting social novelty, extending previous findings of impairment in social recognition in these mutants. Avprlb ${ }^{-1-}$ mice could recognize previously explored objects and remember where they were experienced, but they were impaired in remembering the temporal order of presentation of those objects. Consistent with this finding, Avpr $1 \mathrm{~b}^{-1-}$ mice were also impaired on an object- odor paired associate task that involved a temporal discontiguity between the associated elements. Finally, Avpr $1 \mathrm{~b}^{-1-}$ mice performed normally in learning a set of overlapping odor discriminations and could infer relationships among odors that were only indirectly associated (i.e., transitive inference), indicating intact relational memory. The Avpr $1 b$ is expressed at much higher levels than any other part of the brain in the pyramidal cells of hippocampal CA2 area, a subfield of the hippocampus that has physiological and genetic properties that distinguish it from subfields CA1 and CA3. The combined results suggest that the Avpr1b, perhaps in CA2, may play a highly specific role in social behavior and episodic memory. Because schizophrenia and bipolar disorder are associated with a unique pathology in CA2 and impairments in both social behavior and episodic memory, this animal model could provide insights into the etiology of these disorders.

\section{Introduction}

The vasopressin $1 \mathrm{~b}$ receptor (Avpr1b) plays an important role in the regulation of social behaviors (Wersinger et al., 2002). Mice with a selective deletion of Avpr1b (Avpr1b $\mathrm{b}^{-1-}$ ) are impaired in social recognition, conspecific aggression, and pregnancy block, also known as the Bruce effect (Wersinger et al., 2004, 2007, 2009). However, Avpr1b ${ }^{-/-}$mice display normal mating behavior, olfactory discrimination, prey recognition, and defensive aggression (Caldwell et al., 2008). In contrast to the deficits in social behavior, Avpr1b ${ }^{-1-}$ mice have intact sensorimotor function, exploratory behavior, and spatial memory (Wersinger et al., 2002).

The highest level of brain expression of Avpr1b is in the CA2 subfield of the dorsal hippocampus (Young et al., 2006). Although the structure, function, and physiology of hippocampal subfields CA1 and CA3 have been extensively studied (Leutgeb et al., 2004; Kesner et al., 2005), very little is known about the functional role of the transitional area CA2. Interestingly, schizophre-

Received Nov. 12, 2008; revised Dec. 14, 2008; accepted Jan. 13, 2009.

This work was supported by grants from the Conte Center for Schizophrenia Research-National Institutes of Health and by National Institute of Mental Health Intramural Research Program Grant Z01-MH-002498-20 (W.S.Y.). Thanks to Katherine $0^{\prime}$ Brien for assistance with behavioral training, Will Sauls for skilled mouse husbandry and maintenance of the breeding colony, and Emily Shepard for genotyping. We also thank Dr. Norbert Fortin for comments on this manuscript.

Correspondence should be addressed to Dr. Howard Eichenbaum, Center for Memory and Brain, Boston University, Boston, MA 02215. E-mail: hbe@bu.edu.

DOI:10.1523/JNEUROSCI.5488-08.2009

Copyright $\odot 2009$ Society for Neuroscience $\quad 0270-6474 / 09 / 292676-08 \$ 15.00 / 0$ nia (as well as bipolar disorder) is associated with a selective and significant decrease in the density and number of nonpyramidal cells in CA2 (Benes et al., 1998); additionally, schizophrenic patients exhibit significant deficits in both social behavior (Dickerson et al., 1999; Frank and Landgraf, 2008) and episodic memory, and the latter deficits are specifically associated with hippocampal pathology (Weinberger, 1999; Titone et al., 2004). These findings collectively suggest that the Avpr1b might contribute to memory processes via its role within CA2. The previous reports on knock-out mice link Avpr 1b to social behavior but leave open whether that receptor plays a role in hippocampal-dependent memory function. Here, we specifically explore multiple aspects of hippocampal-dependent episodic memory in the Avpr1b ${ }^{-1-}$ mouse.

\section{Materials and Methods}

Animals. Avprlb heterozygous (Het) (Avprlb ${ }^{+/-}$) mice were provided from the National Institute of Mental Health (NIMH) (Bethesda, MD), as described previously (Wersinger et al., 2002), except that they had been backcrossed into C57BL/6J mice for $>10$ generations. Homozygous

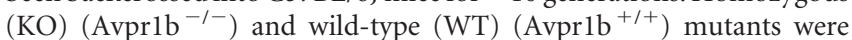
generated from heterozygous breeding pairs at Boston University. At weaning, tail snips were sent to NIMH for genotyping. All animals were maintained on a reverse $12 \mathrm{~h}$ light/dark cycle (9:00 A.M. off; 9:00 P.M. on). Animals were given ad libitum access to food and water, unless otherwise specified in behavioral methods. All animals were male and the group sizes were as follows for each experiment: sociability, $20 \mathrm{WT}$ and

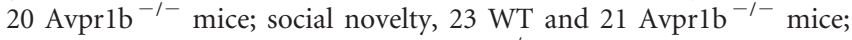
object recognition, $15 \mathrm{WT}$ and $15 \mathrm{Avpr} \mathrm{b}^{-/-}$mice; object-odor associ- 
ation, $8 \mathrm{WT}$ and 7 Avpr1 $1 \mathrm{~b}^{-1-}$ mice; transitive inference, $10 \mathrm{WT}$ and 10 Avprlb ${ }^{-1-}$ mice. Animals were group-housed for the sociability and social novelty experiments; the animals were singly housed for all other experiments. The Institutional Animal Care and Use Committee of Boston University approved the treatment and use of the animals in these experiments.

Social recognition: sociability and preference for social novelty. The social recognition paradigm was adapted from that of Moy et al. (2004). The apparatus was a rectangular plastic box $(40 \mathrm{~cm}$ length $\times 20 \mathrm{~cm}$ width $\times$ $20 \mathrm{~cm}$ height), divided into three areas of equal width by a thin plastic mesh that allowed the animals to explore one another but prevented aggressive interactions (see Fig. $1 \mathrm{~A}$ ). A stimulus animal was placed into one of the outer compartments (left and right placement was counterbalanced). The test animal was then placed into the center compartment and was allowed to investigate both outer compartments. The apparatus was cleaned with $70 \%$ ethanol after each test.

To assess sociability, the test animal was exposed to one compartment containing a familiar littermate and the other compartment was empty. Investigation was measured as the time over a 5 min test in which the animal sniffed each compartment (i.e., when the animal's nose was facing and within $2 \mathrm{~cm}$ the compartment) or climbed the mesh divider. Animals expressing normal sociability are expected to spend a greater amount of time sniffing and climbing the compartment containing the familiar littermate compared with the empty compartment. The score for sociability was calculated as the difference between the investigation time for the compartment containing the familiar littermate and that for the empty compartment.

The day after sociability testing, the animals were assessed on their preference for social novelty. A stimulus animal from a different litter was placed into one compartment and a littermate (different from the animal used in the sociability test) was placed into the other compartment. As in the test for sociability, investigation of each compartment was defined as sniffing and climbing the mesh divider. An animal expressing a normal preference for social novelty will spend a greater amount of time sniffing and climbing the compartment containing the animal from a different litter (novel) compared with the compartment containing the littermate (familiar). The score for social novelty was calculated as the difference between the investigation times for the compartment containing the novel animal and that for the familiar littermate.

Object recognition: "what-where-when" memory for objects. The animals were tested on an episodic memory task that assesses memory for objects ("what"), their locations ("where"), and the order in which they were experienced ("when") (Dere et al., 2005). Animals were first habituated to a rectangular testing apparatus with proximal visual cues on the wall for a $5 \mathrm{~min}$ period ( $40 \mathrm{~cm}$ length $\times 20 \mathrm{~cm}$ width $\times 20 \mathrm{~cm}$ height $)$. On the second day, they were presented with two different objects, to which they would not be reexposed, placed in the northeast and northwest corners of the apparatus, for another $5 \mathrm{~min}$ period. The following day, the animals were given two sample sessions and one test trial (see Fig. $2 \mathrm{~A}$ ). In sample 1 , the animal was allowed $5 \mathrm{~min}$ to explore four identical novel objects (object A; hexagonal weigh boats; VWR) arranged in a triangular shape. After a 50 min delay, in sample 2, the animal explored four new identical objects (object B; paper cups; Sweetheart) arranged in a rectangular shape. After another 50 min delay, the animal was given a test trial in which two copies of object B were placed in the northeast and southwest corners, as in sample 2 ("recent objects"), one copy of object A was placed in the northwest corner as it had been in sample 1 ("stationary old object"), and one copy of object A was newly located to the southeast corner ("displaced old object"). Exploratory behavior was recorded via a webcam (Gigaware) positioned above the testing arena and later scored for investigation time of each object in both sample sessions and the test session. Two researchers blind to the subject's status coded each video. Any animal showing a significant spatial preference, as defined by the greatest amount of exploration occurring at the same location relative to all other locations in both sample and test trials, was removed from analysis, because such behavior indicates that the animal's memory for the objects, their order of presentation, and their location has not mod- ulated its innate preferences. Only one WT mouse and one Avpr1b ${ }^{-1-}$ mouse was removed from the data set for this reason.

Recognition memory for the object ("what") was defined as the difference between the average exploration times for objects A ("old") in sample 1 and objects B ("recent") in sample 2. "Where" memory was defined as the difference between exploration times for the "displaced" object A and the "stationary" object A. "When" memory was defined as the difference between the exploration time for the stationary object A and the average exploration times for objects B (recent).

Object association with a temporal discontiguity. The ability to learn and express memory for a sequence of events was assessed by adapting for mice the object-trace-odor task that Kesner et al. (2005) developed for rats. Animals were placed on food restriction and maintained at $85 \%$ of ad libitum feeding weight. They were shaped over a $3 \mathrm{~d}$ period to dig for chocolate sprinkle rewards buried in sand that filled small plastic cups. Once they were reliably digging, they were given a simple olfactory discrimination of 10 trials across $2 \mathrm{~d}$ to teach them to dig in a cup with a specifically assigned odor. Subsequently, animals were habituated to a Plexiglas runway ( $14 \mathrm{~cm}$ height $\times 14 \mathrm{~cm}$ width) divided into four boxes by guillotine doors: the start box ( $12 \mathrm{~cm}$ long), object box ( $12 \mathrm{~cm} \mathrm{long),}$ trace box ( $12 \mathrm{~cm}$ long), and test box ( $45 \mathrm{~cm}$ long) (see Fig. $3 A$ ). Animals were placed into the start box and allowed to habituate for a few seconds. The first door was opened allowing the animal to enter the second box in which it encountered an object (A or B) mounted to the far wall. Mice were required to explore the object (by sniffing or climbing on it) for at least $3 \mathrm{~s}$. Then the door was lifted, allowing the animals to enter the "trace" box where they were held for a $10 \mathrm{~s}$ delay. Finally, the last door was lifted and the animals were allowed into the test box where they were presented with an odor (1 or 2). Object A was a pink plastic flower (Walgreens) and object B was a yellow plastic Tigger magnet (Disney); odor 1 was coffee (Folger's) and odor 2 was cumin (McCormick; odors were made at $1 \%$ concentration by weight in sand). Animals were only rewarded for digging in the cup with odor 1 if it followed object A and for digging in the cup with odor 2 if preceded by object B ("go" trials); no reward was given for the alternate pairings ("no-go" trials). If a mouse dug within $10 \mathrm{~s}$ in a correctly assigned cup, it received a chocolate sprinkle reward; if a mouse dug in an incorrect cup, the cup was removed and the mouse was left in the box for an additional $10 \mathrm{~s}$ as punishment. Trial type presentation was randomized and each animal received 16 trials per day ( 8 go trials and 8 no-go trials) over six training blocks consisting of 60 trials each. Five animals were run at a time, so that each animal received a 4 min intertrial interval between trials. Latency difference was used as a measure of learning and was defined as the difference between the average latency to dig on no-go and go trials.

After the animals completed the six blocks of training trials, they were tested on a choice version of the task in which, in each test phase, both odors were presented. If a mouse dug in the correct cup, it was given a chocolate sprinkle reward; however, there was no punishment for digging in the incorrect cup. Trial type presentation was randomized and each animal received 16 trials per day, 8 trials with object A and 8 trials with object $\mathrm{B}$, for three test blocks consisting of 60 trials each with a 4 min intertrial interval. Performance on the choice task was measured as percentage correct.

Transitive inference: relational memory for items with overlapping elements. To evaluate the ability to integrate memories and use them flexibly, we adapted for mice the transitive inference task that Dusek and Eichenbaum (1997) developed for rats. Animals were placed on food restriction and maintained at $85 \%$ of ad libitum feeding weight. They were shaped over a $3 \mathrm{~d}$ period to dig for chocolate sprinkle rewards buried in sand that filled small plastic cups. Once they were reliably digging, they were given a simple olfactory discrimination of 10 trials across $2 \mathrm{~d}$ to teach them to dig in a cup with a specifically assigned odor. All odors were made at $1 \%$ concentration by weight in sand.

After the preliminary discrimination problem, animals were trained on a series of overlapping discriminations $[\mathrm{A}+\mathrm{vs} \mathrm{B}-, \mathrm{B}+\mathrm{vs} \mathrm{C}-, \mathrm{C}+\mathrm{vs}$ $\mathrm{D}-$, and $\mathrm{D}+\mathrm{vs} \mathrm{E}-$, where A refers to paprika (CVS), B refers to coffee (Folger's), C refers to basil (McCormick), D refers to cumin (McCormick), and E refers to cocoa (Hershey's); + and - refer to rewarded and nonrewarded odors, respectively] (see Fig. 4A). Animals were trained in 
Table 1. Stages of training for the odor pairs in transitive inference

\begin{tabular}{|c|c|}
\hline Stage & Training schedule \\
\hline 1 & $\begin{array}{l}\text { Day } 1 \\
\text { Session 1: AB (eight trials) } \\
\text { Session 2: BC (eight trials) }\end{array}$ \\
\hline & $\begin{array}{l}\text { Day } 2 \\
\text { Session 1: CD (eight trials) } \\
\text { Session 2: DE (eight trials) }\end{array}$ \\
\hline 2 & $\begin{array}{l}\text { Session } 1: A B \text { and } B C \text { (four trials each) } \\
\text { Session 2: } B C \text { and } D E \text { (four trials each) }\end{array}$ \\
\hline 3 & $\begin{array}{l}\text { Session 1: } A B, B C, C D \text {, and } D E \text { (two trials each) } \\
\text { Session 2: repeat }\end{array}$ \\
\hline 4 & $\begin{array}{l}\text { Session 1: pseudorandom presentation of all pairs: two trials of each pair } \\
\text { Session 2: repeat }\end{array}$ \\
\hline
\end{tabular}

two eight trial sessions per day across four training stages that began with large blocks of trials of the same type and involved progressively more mixing of trial types as described in Table 1. Animals were trained to reach a criterion of $75 \%$ accuracy ( six of eight trials) across 2 consecutive days at each stage of training.

Immediately after reaching criterion on the last training stage, animals were given probe tests for transitive (B vs D) and nontransitive (A vs E) pairs, novel discriminations between items that had not previously been presented together. Four BD and four AE probe tests were intermixed within the training pairs over a $2 \mathrm{~d}$ period, given at trials $3,6,11$, and 14 within the regular 16 trials. A correct judgment (choosing B over D) on the $\mathrm{BD}$ pair required that animals had linked the odor premises so that they could make the inference across the missing overlapping element $\mathrm{C}$. In contrast, a correct judgment of $\mathrm{A}$ over $\mathrm{E}$ could be made without reference to the structure of the odor premises because odor A was always rewarded and odor E was never rewarded; the AE pair served as a control for the presentation of novel pairs. Neither cup is baited during probe trials, and we measured the amount of time the animals spent digging in each cup. These digging times were used to calculate a preference index (PI) (Bunsey and Eichenbaum, 1996). For the B versus D test, $\mathrm{PI}=(\mathrm{B}-$ $\mathrm{D}) /(\mathrm{B}+\mathrm{D})$; for A versus $\mathrm{E}, \mathrm{PI}=(\mathrm{A}-\mathrm{E}) /(\mathrm{A}+\mathrm{E})$.

\section{Results}

\section{Social recognition: sociability and preference for social novelty}

The sociability test assesses the animal's level of social motivation and interest in social interaction. WT mice spent more time investigating the compartment containing the littermate versus the empty compartment, resulting in a difference score of $34.48 \pm$ $6.53 \mathrm{~s}$. In contrast, Avpr $1 \mathrm{~b}^{-1-}$ mice had a difference score of $6.85 \pm 6.63$, which is significantly lower than that for WT mice $\left(t_{(39)}=8.80 ; p=0.005\right)$ (Fig. $\left.1 B\right)$. Furthermore, the social preference for WT mice was significantly different from zero $\left(t_{(39)}=\right.$ 27.85; $p<0.001)$, whereas that for Avpr $1 b^{-l-}$ mice was not $\left(t_{(39)}\right.$ $=1.06 ; p=0.308)$. The average investigation time for the WT mice was $96.92 \pm 5.55 \mathrm{~s}$ for the littermate and $62.44 \pm 3.53 \mathrm{~s}$ for the empty compartment. The average investigation time for the Avpr1b ${ }^{-1-}$ mice was $76.10 \pm 3.73 \mathrm{~s}$ for the littermate and $63.25 \pm 3.93 \mathrm{~s}$ for the empty compartment. Therefore, Avpr1b ${ }^{-1-}$ mice are less attracted to social stimuli than their wild-type littermates.

The social novelty test assesses the animal's social memory as reflected by a preference for investigating a novel conspecific over a familiar one. WT mice spent considerably more time investigating the compartment containing the novel animal versus the compartment containing the familiar littermate, resulting in a difference score of $32.55 \pm 8.22$. In contrast, Avpr1b ${ }^{-1-}$ mice had a difference score of $7.91 \pm 7.57$, which was significantly lower than that of WT mice $\left(t_{(39)}=4.79 ; p=0.034\right)$ (Fig. $1 B$ ). Additionally, the WT mice had preference scores significantly greater than zero $\left(t_{(42)}=14.27 ; p<0.0001\right)$, whereas the Avpr $1 b^{-1-}$ mice did not $\left(t_{(40)}=1.09 ; p=0.302\right)$. The average investigation time for the WT mice was $84.74 \pm 5.13 \mathrm{~s}$ for the novel animal and $52.19 \pm 4.29 \mathrm{~s}$ for the littermate. The average investigation time for the Avpr $1 b^{-1-}$ mice was $74.23 \pm 5.69 \mathrm{~s}$ for the novel animal and $66.31 \pm 3.79 \mathrm{~s}$ for the littermate. Therefore, Avpr $1 b^{-1-}$ mice do not show a preference for a novel animal over that of a familiar littermate, indicating that they did not recognize the familiar animal or were not differentially attracted to a novel conspecific.

Object recognition: "what-where-when" memory for objects Our object recognition test evaluated "what," "when," and "where" memory. "What" memory is reflected by greater exploration time for less recently experienced objects over more recently experienced objects, and is measured as the difference between the average exploration times of the older objects A and the recent objects $\mathrm{B}$. WT mice explored older objects for more time than recent objects, resulting in an average difference score of $42.57 \pm 5.52 \mathrm{~s}$. In comparison, Avpr1b ${ }^{-1-}$ mice had smaller difference scores, averaging $18.31 \pm 6.87 \mathrm{~s}$, which was lower than that of WT mice $\left(t_{(29)}=7.58 ; p=0.01\right)$ (Fig. $\left.2 B\right)$. Nevertheless, the average difference scores for both the WT mice $\left(t_{(29)}=59.43\right.$; $p<0.001)$ and the Avpr1b ${ }^{-1-}$ mice $\left(t_{(29)}=7.11 ; p=0.013\right)$ were greater than zero. The average investigation time for the WT mice was $68.64 \pm 5.25 \mathrm{~s}$ for the old objects and $26.06 \pm 2.98 \mathrm{~s}$ for the recent objects. The average investigation time for the Avpr $1 b^{-1-}$ mice was $51.77 \pm 4.77 \mathrm{~s}$ for the old objects and $34.25 \pm 4.14 \mathrm{~s}$ for the recent objects. Therefore, although Avpr1b ${ }^{-1-}$ mice had weaker "what" memory, both groups showed significant memory for the objects.

"Where" memory refers to the memory for the specific places in which the objects were experienced, reflected in the difference between the average exploration times for the displaced versus the stationary object A. WT mice spent substantially more time investigating the displaced object, reflected in a difference score of $37.76 \pm 7.25 \mathrm{~s}$. Avpr1 $1 \mathrm{~b}^{-1-}$ mice also spent significantly more time exploring the displaced object (difference score of $32.79 \pm$ $7.45 \mathrm{~s}$ ), such that the difference scores of the WT and Avpr $1 \mathrm{~b}^{-1-}$ mice $\left(t_{(29)}=229 ; p=0.636\right)$ did not differ (Fig. $2 B$ ). Furthermore, both WT $\left(t_{(29)}=27.14 ; p<0.001\right)$ and Avpr1b ${ }^{-1-}$ mice $\left(t_{(29)}=19.36 ; p<0.001\right)$ had difference scores significantly greater than zero. The average investigation time for the WT mice was $85.76 \pm 6.46 \mathrm{~s}$ for the displaced object and $47.71 \pm 4.31 \mathrm{~s}$ for the stationary object. The average investigation time for the Avpr $1 \mathrm{~b}^{-1-}$ mice was $69.48 \pm 6.61 \mathrm{~s}$ for the displaced object and $36.69 \pm 4.22 \mathrm{~s}$ for the stationary object. Therefore, the Avpr1b ${ }^{-1-}$ mice had an intact memory for where objects had been experienced.

"When" memory refers to the memory for which of the two objects was experienced earlier, and is measured as the difference between the average exploration times for the stationary older object A and the recent stationary objects B. WT mice had a substantially greater preference for the older stationary object versus the recent objects, reflected in a difference score of $23.69 \pm$ $5.97 \mathrm{~s}$. In contrast, the difference score for the Avpr1b ${ }^{-1-}$ mice was $1.92 \pm 6.55 \mathrm{~s}$, significantly lower than that of WT mice $\left(t_{(29)}\right.$ $=6.03 ; p=0.021$ ) (Fig. $2 B$ ). Furthermore, WT mice demonstrated a preference that is significantly different from zero $\left(t_{(29)}\right.$ 
A

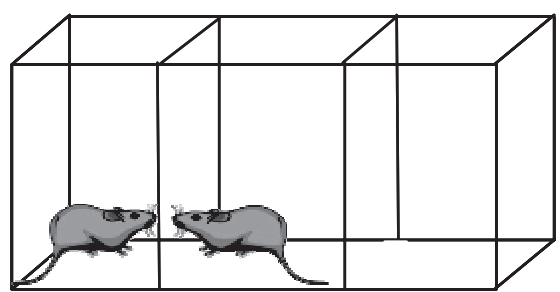

Littermate Subject

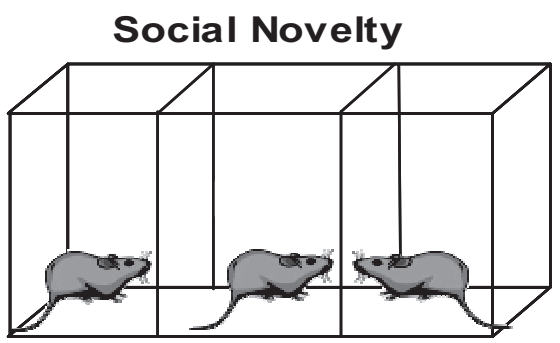

Littermate Subject Novel Animal

\section{Social Novelty}

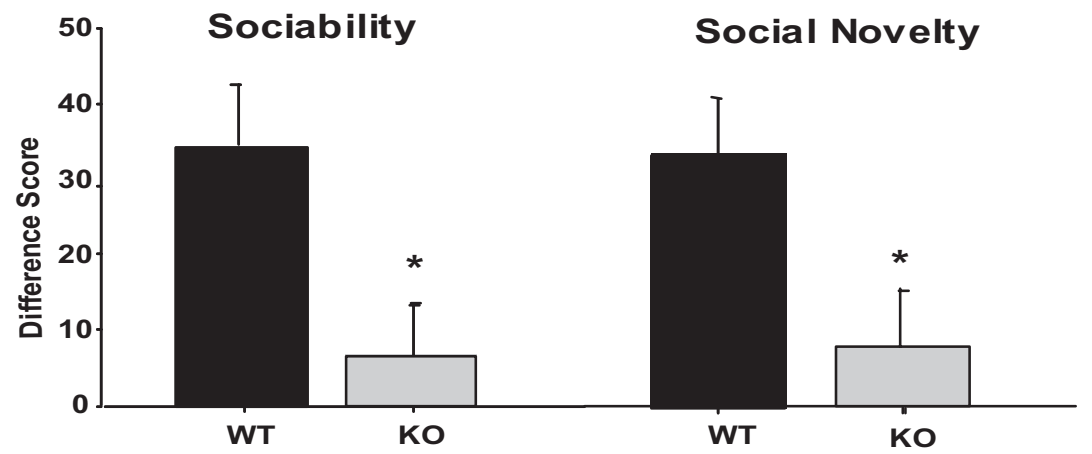

Figure 1. Performance of Avpr $1 b^{-1-}$ and WT mice on social recognition. $\boldsymbol{A}$, Sociability and social novelty task. $\boldsymbol{B}$, A difference score was used to assess preference for a compartment containing a littermate compared with an empty compartment. Avpr $1 b^{-1-}$ mice are deficient in sociability. A difference score was also used to compare the amount of time spent investigating a familiar versus novel animal. Avpr $1 b^{-1-}$ mice do not show a preference for social novelty. ${ }^{*} p<0.05$. Error bars indicate SEM.

\section{A}

Sample One

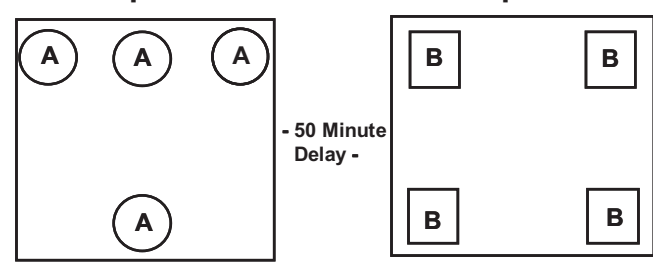

Test

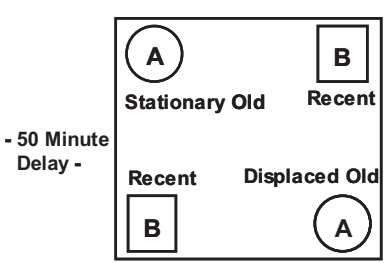

B

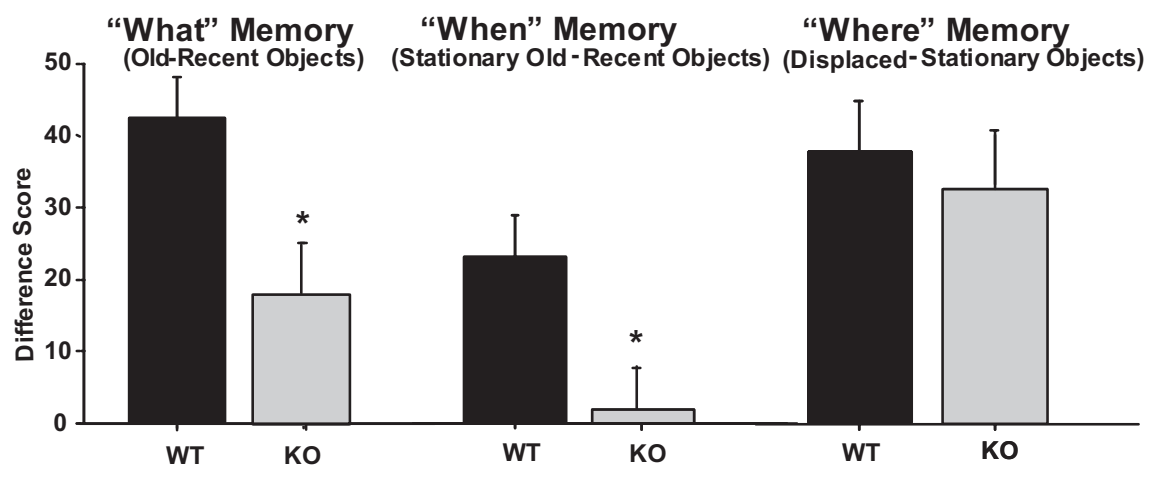

Figure 2. Performance of the Avpr $1 b^{-/-}$and WT mice on object recognition. $A$, "What-where-when" memory task. $\boldsymbol{B}, A$ difference score was used to assess preference for the older versus recent objects ("what" memory). Avpr1b ${ }^{-1-}$ mice display significant "what" memory, but WT mice display a greater preference than the Avpr $1 b^{-1-}$ mice. A difference score was also used to assess preference for the displaced versus the stationary object ("where" memory). Avpr1 $1 \mathrm{~b}^{-1-}$ mice display significant "where" memory. In addition, a difference score was used to assess preference for the older stationary object versus the more recent objects ("when" memory). Avpr $1 b^{-1-}$ mice do not display significant "when" memory. * $p<0.05$. Error bars indicate SEM.
$=15.73 ; p<0.001)$, whereas the Avprlb ${ }^{-1-}$ mice do not $\left(t_{(29)}=0.08 ; p=\right.$ 0.772 ). The average investigation time for the WT mice was $47.71 \pm 4.31 \mathrm{~s}$ for the old object and $27.05 \pm 3.26 \mathrm{~s}$ for the recent objects. The average investigation time for the Avpr1b ${ }^{-1-}$ mice was $36.69 \pm 4.22 \mathrm{~s}$ for the old object and $34.78 \pm 4.49 \mathrm{~s}$ for the recent objects. Therefore, memory for "when" was significantly impaired in Avpr $1 b^{-1-}$ mice, and these mice did not significantly differentiate objects by when they were experienced.

\section{Object association with a}

temporal discontiguity

This task assesses the ability to learn an association between items presented sequentially and separated in time. Avprlb $b^{-/-}$ and WT mice were trained on two objecttrace-odor paired associates and acquisition of the assigned associations was measured by the average latency for responses on the assigned paired associates and that for mismatches on each training block. Both groups learned at a similar rate across the six training blocks (two-way ANOVA: group, $F_{(1,89)}=2.51 ; p=0.117$; trial block, $F_{(5,80)}=2.32 ; p=0.051$; group by trial block interaction, $F_{(5,80)}=2.18 ; p=0.064$ ) (Fig. 3B); Avpr1b ${ }^{-1-}$ mice were significantly impaired on only training block 2 compared with the WT mice $\left(t_{(13)}=6.64\right.$; $p=0.023)$. Overall performance on the training trials was not significantly different between the two groups $\left(t_{(13)}=1.32\right.$; $p=0.270)$.

Although both groups acquired the training pairs at a similar rate, the Avpr $1 \mathrm{~b}^{-1-}$ mice displayed significant impairment on the choice task (two-way ANOVA: group, $F_{(1,44)}=54.03 ; p \leq 0.001$; test block, $F_{(2,44)}=0.15 ; p=0.857$; group by pair type interaction, $F_{(2,44)}=3.91 ; p=$ 0.028) (Fig. 3C). The performance of Avpr $1 b^{-1-}$ mice compared with the WT mice was significantly lower across all three test blocks (block 1: $t_{(13)}=8.31 ; p=0.013$; block 2: $t_{(13)}=20.05 ; p=0.001$; block 3: $\left.t_{(13)}=27.57 ; p<0.001\right)$. Furthermore, WT mice performed significantly better than chance on all three test blocks (test block 1: $t_{(14)}=21.70 ; p<0.001$; test block 2: $t_{(14)}=$ $35.48 ; p<0.001$; test block $3: t_{(14)}=21.46$; $p<0.001$ ), whereas the Avprlb ${ }^{-1-}$ mice did not perform significantly better than chance and actually performed significantly lower than chance levels on test block 3 (test block $1: t_{(13)}=0.14 ; p=0.712$; test block 2: $t_{(13)}=0.77 ; p=0.397$; test block $\left.3: t_{(13)}=9.28 ; p=0.01\right)$. There was no difference in performance on the individual paired associates (object A or object 

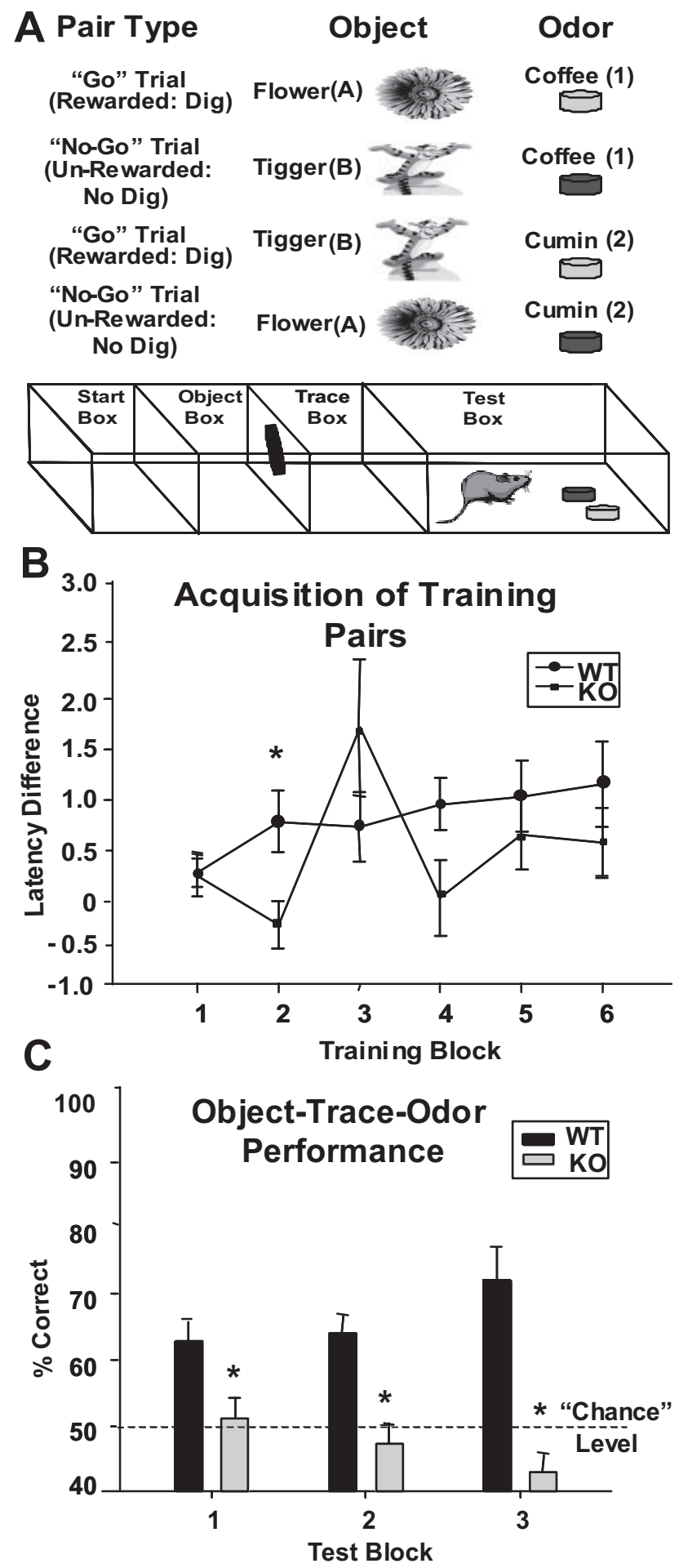

Figure 3. Performance of Avpr $1 b^{-1-}$ and WT mice on object associations with a temporal discontiguity. $A, 0$ bject-trace- odor task. $B$, Mean latency difference between the no-go versus go trials was used as a measure of acquisition of the training pairs. Although Avpr $1 b^{-1-}$ and WT mice learned the training pairs at an equivalent rate, the Avpr $1 b^{-I-}$ mice displayed impairment at block 2. C, Percentage correct was used to assess performance on the choice test. Avpr $1 \mathrm{~b}^{-I-}$ mice are significantly impaired at recalling object- odor paired associates with a 10 s delay. ${ }^{*} p<0.05$. Error bars indicate SEM.

B), but there was a significant effect of group on overall performance on the choice task (two-way ANOVA: group, $F_{(1,29)}=$ $14.17 ; p=0.001$; pair type, $F_{(1,29)}=0.96 ; p=0.355$; group by test block interaction, $\left.F_{(1,29)}=2.82 ; p=0.105\right)$. These results indi- cate that Avpr1b ${ }^{-1-}$ mice can acquire the appropriate response across many repetitions of the object-trace-odor sequences, but they cannot flexibly use this information to recall which of two odors follows the associated object.

Transitive inference: relational memory for items with overlapping elements

The transitive inference task assesses the ability of animals to learn a series of paired associations that share common elements, and subsequently to use these memories to infer indirect relationships between the elements that were never experienced together. Avpr $1 b^{-1-}$ and WT mice successfully learned the four odor pair discriminations and there was no difference between the two groups in the number of days required to reach criterion on the four stages of training (two-way ANOVA: group, $F_{(1,79)}=0.06$; $p=0.795$; stage, $F_{(3,19)}=0.37 ; p=0.772$; group by stage interaction, $\left.F_{(3,79)}=0.11 ; p=0.953\right)$. This indicates that Avpr $1 \mathrm{~b}^{-1-}$ mice have an intact ability to learn a series of overlapping odor discriminations at a rate that is equivalent to their wild-type littermates.

Although both groups performed at comparable levels on the odor pairs, there were differences in performance on the trained odor pairs when tested along with the probe pairs. Avpr1b ${ }^{-1-}$ mice showed an atypical pattern of performance compared with their wild-type littermates (two-way ANOVA: group, $F_{(1,79)}=$ $0.54 ; p=0.461$; pair type, $F_{(3,79)}=5.56 ; p=0.002$; probe pair by pair type interaction, $F_{(3,79)}=2.35 ; p=0.079$ ) (Fig. $4 B$ ). Specifically, WT mice performed better on the DE pair than each of the other pairs $[\mathrm{AB}, 66.67 \pm 6.08 \%$; $\mathrm{BC}, 61.67 \pm 6.11 \%$; $\mathrm{CD}, 63.33 \pm$ 6.93\%; DE, $91.67 \pm 4.47 \%$; one-way ANOVA: $F_{(3,39)}=5.53 ; p=$ 0.003 ; $\mathrm{AB}$ vs $\mathrm{DE}(p=0.005), \mathrm{BC}$ vs $\mathrm{DE}(p=0.001), \mathrm{CD}$ vs $\mathrm{DE}$ $(p=0.002)]$. Compared with the other pairs, the Avpr1b ${ }^{-1-}$ mice performed significantly better on the CD pair and there was a significant difference between $\mathrm{CD}$ versus $\mathrm{BC}$ and $\mathrm{DE}$ versus $\mathrm{BC}$ $[\mathrm{AB}, 75.00 \pm 5.69 \%$; BC, $58.33 \pm 7.95 \%$; CD, $83.33 \pm 4.96 \%$; DE, $80.00 \pm 7.77 \%$; one-way ANOVA: $F_{(3,39)}=2.72 ; p=0.059$, BC vs $\mathrm{CD}(p=0.013), \mathrm{BC}$ vs DE $(p=0.029)]$ Avpr1b $^{-1-}$ and WT mice differed in performance only on the CD pair and no other pair type $\left(\mathrm{CD}: t_{(19)}=5.491 ; p=0.031\right)$.

In the critical probe tests, both Avpr1b ${ }^{-1-}$ and WT mice showed a significant preference for B over D and for A over E, indicating intact relational memory. WT mice significantly preferred $\mathrm{B}$ over $\mathrm{D}\left(\mathrm{PI}=0.35 \pm 0.13 ; t_{(78)}=26.39 ; p<0.001\right)$ and A over $\mathrm{E}\left(\mathrm{PI}=0.82 \pm 0.07 ; t_{(78)}=467.60 ; p<0.001\right)$ at levels significantly different from chance (Fig. $4 C$ ). Avpr1b ${ }^{-1-}$ mice performed at nearly identical levels on the $\mathrm{BD}(\mathrm{PI}=0.35 \pm 0.12$; $\left.t_{(78)}=30.73 ; p<0.001\right)$ and $\mathrm{AE}$ probe tests $(\mathrm{PI}=0.077 \pm 0.06$; $\left.t_{(78)}=568.28 ; p<0.001\right)$ at levels significantly different from chance. There was no difference in performance between the two groups on the BD probe tests $\left(t_{(79)}=0.00 ; p=0.991\right)$ or the $\mathrm{AE}$ probe tests $\left(t_{(79)}=1.17 ; p=0.282\right)$. In addition, the total amount of time spent digging in all probe cups was equivalent, showing that the animals were just as motivated and displayed no impairments in motivation or activity levels $\left(t_{(318)}=0.85 ; p=0.355\right)$.

\section{Discussion}

The present results indicate that the Avpr1b plays an important role in social preferences and episodic memory, most specifically in the temporal components of the object recognition and paired associate tasks. We found that Avpr1b ${ }^{-1-}$ mice are deficient in sociability and fail to show a normal preference for social novelty. Avpr $1 b^{-1-}$ mice are also impaired in remembering the order of a sequence of objects and fail to remember which of two odors are 
A

\section{Odor Pairs}

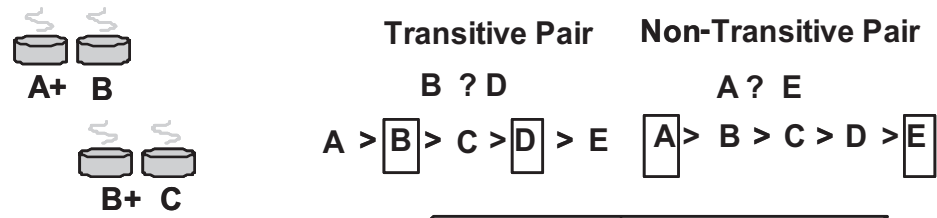

\section{Probe Tests}
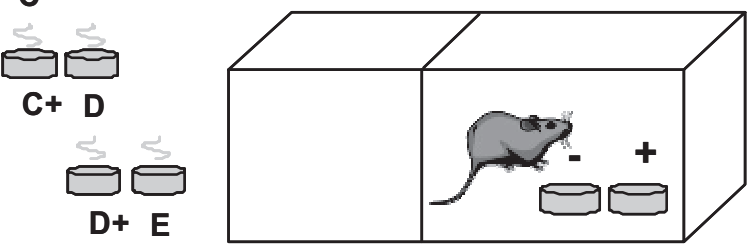

B

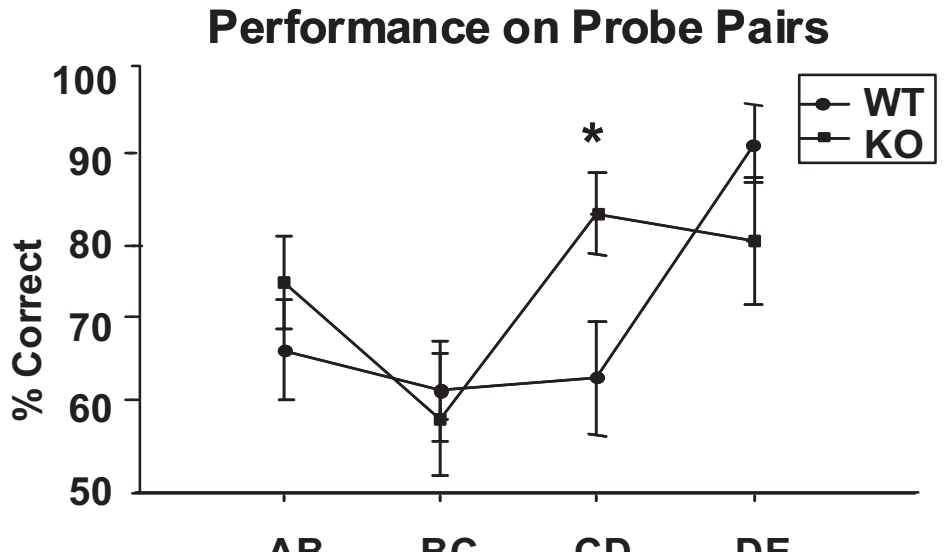

$\mathrm{AB}$

C

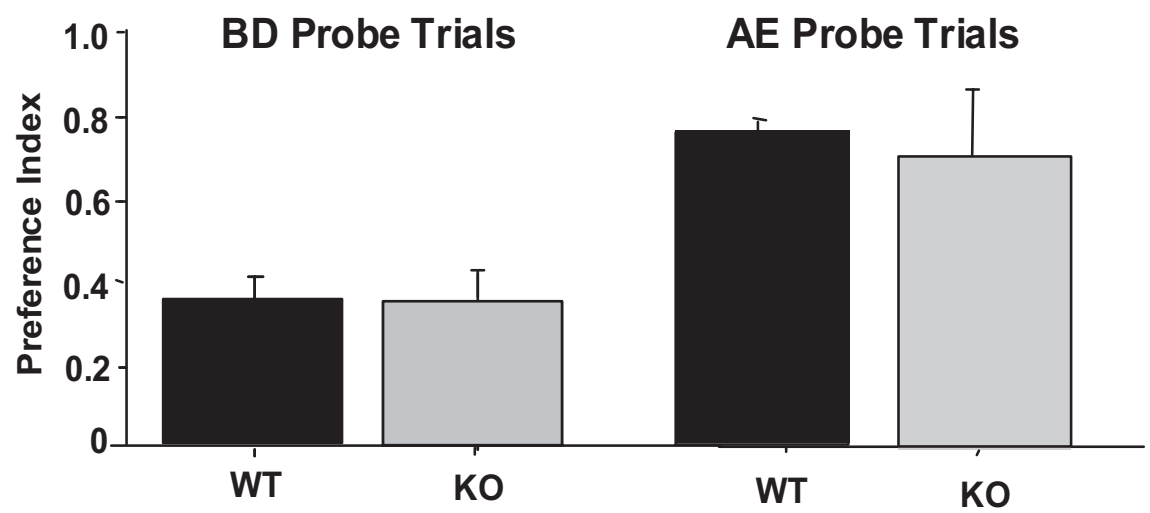

Figure 4. Performance of Avpr $1 b^{-1-}$ and WT mice on relational memory. $A$, Transitive inference task. $\boldsymbol{B}$, Percentage correct was used to assess performance on the training pairs during the transitive inference test. Avpr $1 b^{-1-}$ displayed an atypical pattern of performance on the odor pairs, performing best on the $C D$ pair. $C$, A preference index was used to assess the amount of time spent digging in B versus D ("transitive pair") and A versus $E$ ("nontransitive pair") on the probe tests. Avpr $1 b^{-l-}$ mice performed successfully on both critical probe tests, indicating intact relational memory. ${ }^{*} p<0.05$. Error bars indicate SEM.
These data suggest that Avpr1b ${ }^{-1-}$ mice are specifically impaired in memory requiring the ability to compare events that occurred at different times.

\section{Impaired social recognition}

Our results indicate that the Avpr1b ${ }^{-1-}$ mice display impairment in detecting or preferring social novelty. Previous studies have shown that Avpr1b ${ }^{-1-}$ mice display impairments in habituating to a female stimulus after repeated presentations (Wersinger et al., 2002). Avpr1b ${ }^{-1-}$ mice are able to distinguish male and female urine, as well as perform a hidden cookie test. Additionally, neural activation in the main and accessory bulbs does not differ between Avprlb ${ }^{-1-}$ and WT mice after exposure to a novel animal (Wersinger et al., 2004). Thus, it does not appear that these deficits are attributable to olfactory dysfunction, but instead lie in the interpretation or expression of behavior based on these signals.

It has been proposed that these olfactory-mediated deficits are attributable to a failure of associating a social encounter with the context in which it occurs (Caldwell et al., 2008). This view is supported by work showing decreased social investigation and recognition in fimbria-transected rats, as well as with hippocampal lesions in mice (Maaswinkel et al., 1997; Kogan et al., 2000).

Impaired object recognition and memory for when events occurred The object recognition task allows for the simultaneous assessment of memory for "what," "where," and "when," three core features of episodic memory that are known to be dependent on the integrity of the hippocampus (Ergorul and Eichenbaum, 2004). We found that Avpr1b mice display abnormalities on the "what" (object) and "when" (temporal order) components of the task, in contrast with intact performance for "where" (place) memory. The results on "where" memory are consistent with previous findings of proficient performance on the Morris water maze (Wersinger et al., 2002), suggesting that spatial memory is preserved in these animals. Because the Avpr1b ${ }^{-1-}$ mice displayed a significant preference for the object memory component, but not the temporal order component, we can conclude that these animals are most impaired in memories that involve a strong temporal component. associated with a preceding object across a delay. In contrast, although Avpr1b ${ }^{-1-}$ mice show significant, albeit impaired object recognition, they can remember where objects were experienced and perform normally on a test of relational memory.
Impairment in expressing memory for associations that involves a temporal discontiguity

To confirm and extend our findings on temporal processing in the object recognition study, we tested the Avpr $1 b^{-1-}$ mice on an 
associative task that requires the ability to bridge associations across a temporal gap. We found that Avpr1b ${ }^{-1-}$ mice are impaired in associative memory when the associated items are separated by a time delay. This suggests that Avpr1b ${ }^{-1-}$ mice fail to integrate information across a temporal delay or to successfully recall associations that are linked across a delay.

\section{Intact relational memory}

We were also interested in determining whether Avpr1b ${ }^{-1-}$ mice could perform a hippocampal-dependent associative olfactory task without a temporal component. The medial temporal lobe has been shown to be involved in relational memory, the ability to bind together overlapping items to recreate the full episode (Bunsey and Eichenbaum, 1996; Buckmaster et al., 2004; Heckers et al., 2004). The Avpr1b ${ }^{-1-}$ mice learned the overlapping odor pairs as readily as their wild-type littermates and performed at comparable levels on both the transitive and nontransitive probe tests, demonstrating intact performance on a test of relational memory. Interestingly, they displayed an atypical pattern of performance on the trained odor pairs at the time of probe testing. Although the cause of this difference in pattern of performance is not clear, the findings suggest that Avpr $1 b^{-1-}$ mice may be using a different strategy than WT mice to organize their memories for the odor series.

\section{Are the memory deficits in Avpr $1 b^{-1-}$ mice attributable to dysfunction of hippocampal area CA2?}

The highest expression of Avpr1b is in area CA2, suggesting that dysfunction within this area may underlie the deficits in memory more so than other circuitries that involve this vasopressin receptor. Furthermore, Avpr1b is only one of many receptors within CA2, and deletion of this receptor may not eliminate all contributions of CA2 to social behavior or memory. Thus, although it is possible that Avpr1b elsewhere in the brain is the cause of the memory deficits observed here, and a complete ablation of CA2 might have a broader impact on hippocampal-dependent memory, the present findings suggest that the involvement of Avpr $1 \mathrm{~b}$ in CA2 may result in a highly selective contribution to memory. Other studies have shown that lesions affecting all hippocampal subfields produce deficits in memory for objects (Broadbent et al., 2004) (but see O'Brien et al., 2006), for where they are located (Gilbert and Kesner, 2002), for their temporal order (Fortin et al., 2002), and for their organization in support of transitive inference (Dusek and Eichenbaum, 1997). The present results suggest that CA2, and in particular Avpr1b within CA2, may play a more restricted role in memory, selective to temporal processing that supports episodic memory. Of additional possible relevance, rats with damage to area CA1, but not CA3, are impaired in temporal order memory in the object-trace-odor association task (Kesner et al., 2005). Additional studies may clarify whether CA2 is performing this function independently or in conjunction with area CA1, because lesions to CA1 can sometimes damage cells in CA2 as well. Alternatively, whereas in the study by Kesner et al. (2005) the impairment was observed during the acquisition of the task, in the current study Avpr1b ${ }^{-1-}$ mice acquired the associations and failed only on the subsequent choice test. Therefore, it is possible that CA1 can support acquisition of object-trace-odor sequences, but the expression of these temporally discontinuous associations in a choice test requires CA2 function.

Additional evidence suggests a mechanism by which CA2 may contribute in a specific way to temporal memory processing. Area CA2 of the hippocampus contains several structural and physiological characteristics that differentiate it from CA1 and CA3. It has a distinctive genetic composition and physiological properties that make it a very unique region of the hippocampus (Mercer et al., 2007; Zhao et al., 2007). One specific property was demonstrated by a study using optical recording to image the signal propagation in the hippocampus after stimulation of the dentate gyrus revealed two different signal pathways of signal propagation (Sekino et al., 1997). In the faster signal propagation pathway, activity flows directly from CA3 to CA1; however, in the slower signal propagation pathway, signals are generated first in CA2, persist, and then spread into CA1. These findings indicate that CA2 may be playing an important role in modulating the input of delayed information to CA1. These data provide a possible explanation for our behavioral findings indicating that Avpr $1 b^{-1-}$ animals are impaired at temporal processing in episodic memory. The lack of Avpr $1 \mathrm{~b}$ in these animals may result in a disruption of the function of the CA2 area, which normally is providing important input to CA1 about information of slower time signals, as reflected by the inability to perform memory tasks that require maintenance of a memory signal over a delay period.

Finally, the present data reveal that Avpr $1 b^{-/-}$mice display impairments in both social behavior and specific components of episodic memory that are also disrupted in schizophrenia. This parallel suggests that disruption of CA2 function may provide significant insight into both the etiology of this disorder and about the role of Avprlb in a key aspect of episodic memory.

\section{References}

Benes F, Kwok E, Vincent SL, Todtenkopf M (1998) A reduction of nonpyramidal cells in sector CA2 of schizophrenics and manic depressives. Biol Psychiatry 44:88-97.

Broadbent NJ, Squire LR, Clark RE (2004) Spatial memory, recognition memory, and the hippocampus. Proc Natl Acad Sci USA 101:14515-14520.

Buckmaster CA, Eichenbaum H, Amaral DG, Suzuki WA, Rapp PR (2004) Entorhinal cortex lesions disrupt the relational organization of memory in monkeys. J Neurosci 24:9811-9825.

Bunsey M, Eichenbaum H (1996) Conservation of the hippocampal memory function in rats and humans. Nature 379:255-257.

Caldwell HK, Wersinger SR, Young WS 3rd (2008) The role of the vasopressin $1 \mathrm{~b}$ receptor in aggression and other social behaviors. Prog Brain Res 170:65-72.

Dere E, Huston J, De Souza Silva M (2005) Episodic-like memory in mice: simultaneous assessment of object, place and temporal order memory. Brain Res Protoc 16:10-19.

Dickerson F, Boronow J, Ringel N, Parente F (1999) Social functioning and neurocognitive deficits in outpatients with schizophrenia: a 2-year follow-up. Schizophr Res 37:13-20.

Dusek JA, Eichenbaum H (1997) The hippocampus and memory for orderly stimulus relations. Proc Natl Acad Sci U S A 94:7109-7114.

Ergorul C, Eichenbaum H (2004) The hippocampus and memory for "what," "where," and "when." Learn Mem 11:397-405.

Fortin NJ, Agster KL, Eichenbaum HB (2002) Critical role of the hippocampus in memory for sequences of events. Nat Neurosci 5:458-462.

Frank E, Landgraf R (2008) The vasopressin system-from antidiuresis to psychopathology. Eur J Pharm 583:226-242.

Gilbert PE, Kesner RP (2002) Role of the rodent hippocampus in pairedassociate learning involving associations between a stimulus and a spatial location. Behav Neurosci 116:63-71.

Heckers S, Zalesak M, Weiss AP, Ditman T, Titone D (2004) Hippocampal activation during transitive inference in humans. Hippocampus 14:153-162.

Kesner RP, Hunsaker MR, Gilbert PE (2005) The role of CA1 in the acquisition of an object-trace-odor paired associate task. Behav Neurosci 119:781-786.

Kogan JH, Frankland PW, Silva AJ (2000) Long-term memory underlying hippocampus-dependent social recognition in mice. Hippocampus 10:47-56

Leutgeb S, Leutgeb JK, Treves A, Moser MB, Moser EI (2004) Distinct en- 
semble codes in hippocampal areas CA3 and CA1. Science 305:1295-1298.

Maaswinkel H, Gispen WH, Spruijt BM (1997) Executive function of the hippocampus in social behavior in the rat. Behav Neurosci 111:777-784.

Mercer A, Trigg HL, Thomson AM (2007) Characterization of neurons in the CA2 subfield of the adult rat hippocampus. J Neurosci 27:7329-7338.

Moy SS, Nadler JJ, Perez A, Barbaro RP, Johns JM, Magnuson TR, Piven J, Crawley JN (2004) Sociability and preference for social novelty in five inbred strains: an approach to assess autistic-like behavior in mice. Genes Brain Behav 3:287-302.

O'Brien N, Lehmann H, Lecluse V, Mumby DG (2006) Enhanced context dependency of object recognition in rats with hippocampal lesions. Behav Brain Res 170:156-162.

Sekino Y, Obata K, Tanifuji M, Mizuno M, Murayama J (1997) Delayed signal propagation via CA2 in rat hippocampal slices revealed by optical recording. J Neurophysiol 78:1662-1668.

Titone D, Ditman T, Holzman P, Eichenbaum H, Levy D (2004) Transitive inference in schizophrenia: impairments in relational memory organization. Schizophr Res 68:235-247.

Weinberger D (1999) Cell biology of the hippocampal formation in schizophrenia. Biol Psychiatry 45:395-402.
Wersinger SR, Ginns EI, O'Carroll AM, Lolait SJ, Young WS 3rd (2002) Vasopressin V1b receptor knockout reduces aggressive behavior in male mice. Mol Psychiatry 7:975-984.

Wersinger SR, Kelliher KR, Zufall F, Lolait SJ, O'Carroll AM, Young WS 3rd (2004) Social motivation is reduced in vasopressin $1 \mathrm{~b}$ receptor null mice despite normal performance in an olfactory discrimination task. Horm Behav 46:638-645.

Wersinger SR, Caldwell HK, Christiansen M, Young WS 3rd (2007) Disruption of the vasopressin $1 \mathrm{~b}$ receptor gene impairs the attack component of aggressive behavior in mice. Genes Brain Behav 6:653-660.

Wersinger SR, Temple JL, Caldwell HK, Young WS 3rd (2008) Inactivation of the oxytocin and the vasopressin (Avp) $1 \mathrm{~b}$ receptor genes, but not the Avp la receptor gene, differentially impairs the Bruce effect in laboratory mice (Mus musculus). Endocrinology 149:116-121.

Young WS, Li J, Wersinger SR, Palkovits M (2006) The vasopressin 1b receptor is prominent in the hippocampal area CA2 where it is unaffected by restraint stress or adrenalectomy. Neuroscience 143:1031-1039.

Zhao M, Choi YS, Obrietan K, Dudek SM (2007) Synaptic plasticity (and the lack thereof) in hippocampal CA2 neurons. J Neurosci 27:1202512032 . 